\title{
A Single-Symbol-Decodable Space-Time Block Code with Full Rate and Low Peak-to-Average Power Ratio
}

\author{
Mohanned O. Sinnokrot and John R. Barry
}

School of ECE, Georgia Institute of Technology, Atlanta, GA 30332 USA, \{mohanned.sinnokrot@, barry@ece\}.gatech.edu

\begin{abstract}
Three desirable properties of a four-antenna spacetime block code are full rate, full diversity, and single-symbol decodability. Previously reported space-time codes that achieve all three properties do so at the expense of the peak-to-average power ratio (PAPR). A fourth desirable property of a space-time block code is that its PAPR be the same as that of the underlying quadrature-amplitude modulation alphabet. In this paper we introduce space-time codes for three and four transmit antennas that achieve all four properties; these codes use a diversity technique based on constellation stretching. Numerical results for quasistatic Rayleigh-fading channels show that the proposed codes are comparable in SNR performance to the best-performing single-symbol decodable space-time codes for three and four transmit antennas.
\end{abstract}

Keywords - transmit diversity, quasiorthogonal codes.

\section{INTRODUCTION}

The orthogonal space-time block codes of Alamouti [1] and Tarokh et al. [2] attain full diversity with low decoding complexity, but they suffer a rate loss when there are more than two transmit antennas. A quasiorthogonal code relaxes the orthogonality constraint to enable full rate, at the expense of an increase in decoding complexity. For example, quasiorthogonal codes for four antennas were proposed independently by Jafarkhani [3], Tirkkonen-Boarin-Hottinen [4] and PapadiasFoschini [5]; these full-rate codes have two drawbacks: they are not full diversity, and they require pair-wise complex symbol decoding. The first drawback can be eliminated by constellation rotation. For example, full-rate and full-diversity quasiorthogonal codes with rotation were proposed by Tirkkonen [6], Sharma-Papadias [7] and Su-Xia [8]. While these quasiorthogonal codes outperform orthogonal codes at all spectral efficiencies for four transmit antennas, they still require pair-wise complex symbol decoding.

Recent work has shown that low decoding complexity is possible even with nonorthogonal codes. For example, a combination of constellation rotation and coordinate interleaving was proposed by Yuen et al. [9] and Khan-Rajan [10] to achieve full rate and full diversity for four transmit antennas with ML decoding that requires only pair-wise

This research was supported in part by a grant from Texas Instruments. decoding of real symbols. Since a pair of real symbols defines a single complex symbol, these codes are said to be single-symbol decodable.

Another important property of a space-time block code is its peak-to-average power ratio (PAPR). The PAPR is important for several reasons. First, a high-PAPR signal is susceptible to clipping and other nonlinear distortion by the power amplifier, leading to detection errors as well as out-of-band interference. Second, power consumption of the power amplifier depends mainly on the peak power rather than average power, and hence, high PAPR results in high power consumption. Finally, the transmission of a signal with high PAPR requires the use of a power amplifier with large back off, resulting in inefficient, bulky and expensive power amplifiers [11].

In this paper, we present single-symbol-decodable spacetime block codes based on the coordinate interleaving technique of [9][10] but coupled with constellation stretching rather than constellation rotation. The new code is not only single-symbol decodable, it also maintains the same PAPR as the underlying QAM alphabet. While this paper was under review, we discovered that the concept of constellation stretching was proposed previously and independently by Marsch et al. in [12], albeit with a different motivation and construction. Nevertheless, our paper is unique in at least two respects: we derive the optimal stretching factor for maximizing the coding gain, and we quantify the PAPR benefits of the proposed code, which is the main motivation behind our construction.

The remainder of the paper is organized as follows. Section II presents the system model, relevant definitions, and a brief overview of existing single-symbol-decodable space-time block codes. Section III describes the proposed code along with its decoder, and compares it to existing codes. Section IV presents numerical results and Section V concludes the paper.

\section{SySTEM MODEL AND PRIOR SSD CODES}

\section{A. System Model and Definitions}

We consider a transmitter with $N$ antennas transmitting $K$ complex information symbols over $T$ symbol periods, so that the rate- $K / T$ space-time block code is represented by a $T \times N$ matrix $\mathbf{X}$. Letting $y_{i}$ denote the sample received at time $i$ by a receiver with a single antenna, we can write: 


$$
\left[y_{1}, \ldots y_{T}\right]^{T}=\mathbf{X h}+\mathbf{w},
$$

where $\mathbf{h}$ is a vector of channel gains between the $N$ transmit antennas and the receive antenna, and where $\mathbf{w}$ is the complex Gaussian noise with i.i.d. $\mathcal{C N}\left(0, N_{0}\right)$ entries. We assume a quasistatic Rayleigh-fading channel, so that the entries of $\mathbf{h}$ are i.i.d. $\mathcal{C N}(0,1)$ and constant over the duration of the $T$ symbol periods. The results are easily extended to an arbitrary number of receive antennas.

The PAPR for the $m$-th transmit antenna of a space-time code is [13]:

$$
P A P R_{m}=\frac{\max _{t \in\{1, \ldots, T\}}\left|X_{t, m}\right|^{2}}{T^{-1} \sum_{t \in\{1, \ldots, T\}} E\left(\left|X_{t, m}\right|^{2}\right)},
$$

where the maximum and the expectation operators are taken over all possible codeword matrices. In all the space-time codes considered in this paper, the PAPR is the same for all the antennas, so that the subscript on PAPR in (2) may be dropped. The coding gain of a full-diversity space-time code is [10]:

$$
\Gamma=\min _{\mathbf{X} \neq \tilde{\mathbf{X}}} \operatorname{det}\left((\mathbf{X}-\tilde{\mathbf{X}})^{*}(\mathbf{X}-\tilde{\mathbf{X}})\right)^{1 / N},
$$

where the minimum is taken over all distinct codeword matrices $\mathbf{X} \neq \tilde{\mathbf{X}}$

\section{B. Single-Symbol-Decodable Space-Time Codes}

Single-symbol-decodable space-time bock codes for four transmit antennas were proposed in [9] and [10]. In both cases the encoder decomposes into a concatenation of three steps, as shown in Fig. 1. The encoder starts with a vector $\mathbf{x} \in \mathcal{A}^{4}$ of information symbols chosen from a conventional $M$-ary QAM alphabet $\mathcal{A}$. The first step is to distort the alphabet in some way; the codes of [9] and [10] rotate each alphabet by an angle of $\phi$, producing $\mathbf{a} \in e^{j \phi} \mathbf{x}$, as shown in Fig. 2-(b). The purpose of the rotation is to ensure full diversity. The second step is to interleave the coordinates of $\mathbf{a}$, yielding $\mathbf{s}=\Pi(\mathbf{a})$. The interleavers of [9] and [10] act on the real and imaginary parts separately, so that $\left[s_{1}^{R}, s_{1}^{I}, \quad s_{2}^{R}, s_{2}^{I}, s_{3}^{R}, s_{3}^{I}\right.$, $\left.s_{4}^{R}, s_{4}^{I}\right]=\left[a_{1}^{R}, a_{1}^{I}, a_{2}^{R}, a_{2}^{I}, a_{3}^{R}, a_{3}^{I}, a_{4}^{R}, a_{4}^{I}\right] \Pi$, where $\Pi$ is an $8 \times 8$ permutation matrix (so that its columns are a permutation of the columns of the identity matrix), and where $s_{i}^{R}$ and $s_{i}^{I}$ denote the real and imaginary parts, respectively, of $s_{i}$. The interleaver ensures full diversity without sacrificing

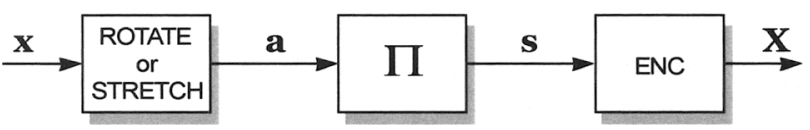

Fig. 1. Encoding architecture. single-symbol decodability. The final step is to encode $\mathbf{s}$ using a conventional space-time block encoder $\mathbf{G}(\cdot)$, yielding $\mathbf{X}=$ $\mathbf{G}(\mathbf{s})$.

In terms of Fig. 1, the Khan-Rajan code of [10] is specified by $\phi=\frac{1}{2} \tan ^{-1}(2), \Pi=\left[\boldsymbol{e}_{1}, \boldsymbol{e}_{6}, \boldsymbol{e}_{3}, \boldsymbol{e}_{8}, \boldsymbol{e}_{5}, \boldsymbol{e}_{2}, \boldsymbol{e}_{7}, \boldsymbol{e}_{4}\right]$, where $\boldsymbol{e}_{i}$ is the $i$-th column of the $8 \times 8$ identity matrix, and:

$$
\begin{array}{cc} 
& \mathbf{G}(\mathbf{s})=\sqrt{2}\left[\begin{array}{cc}
\mathbf{A}\left(s_{1}, s_{2}\right) & 0 \\
0 & \mathbf{A}\left(s_{3}, s_{4}\right)
\end{array}\right], \\
\text { where } & \mathbf{A}\left(s_{1}, s_{2}\right)=\left[\begin{array}{cc}
s_{1} & s_{2} \\
-s_{2}^{*} & s_{1}^{*}
\end{array}\right]
\end{array}
$$

is the Alamouti space-time code [1]. The constant $\sqrt{2}$ ensures that the average transmit energy per transmitted symbol (namely the denominator in (2)) is identical to the energy of the underlying alphabet $\mathcal{A}$. As noted in [9], a drawback of this code is that half of its entries are zero, which leads to a high PAPR.

The Yuen-Guan-Tjhung code of [9] can also be defined in terms of Fig. 1, with a rotation angle of $\phi=\pi / 4-\frac{1}{2} \tan ^{-1}(2)$, an interleaver of $\Pi=\left[\boldsymbol{e}_{1}, \boldsymbol{e}_{5}, \boldsymbol{e}_{3}, \boldsymbol{e}_{7},-\boldsymbol{e}_{2}, \boldsymbol{e}_{6},-\boldsymbol{e}_{4}, \boldsymbol{e}_{8}\right]$, and:

$$
\mathbf{G}(\mathbf{s})=\left[\begin{array}{ll}
\mathbf{A}\left(s_{1}, s_{2}\right) & \mathbf{A}\left(s_{3}, s_{4}\right) \\
\mathbf{A}\left(s_{3}, s_{4}\right) & \mathbf{A}\left(s_{1}, s_{2}\right)
\end{array}\right] .
$$

\section{Code Construction AND Decoding}

\section{A. Code Construction and Optimization}

We propose a new code based on the same architecture of Fig. 1 but differing in an important way from [9] and [10]: rather than distorting the alphabet by a rotation, so that $a_{i}=$ $e^{j \phi} x_{i}$, we instead propose to distort it by stretching it vertically, using:

$$
a_{i}=\sqrt{\frac{2}{1+K^{2}}}\left(K x_{i}^{R}+j x_{i}^{I}\right),
$$

where $K \in(0,1]$ is a stretching parameter to be specified later. The constant $\sqrt{2 /\left(1+K^{2}\right)}$ ensures that the stretched alphabet has the same energy as the original. Scaling the real and imaginary parts of the symbol by different amounts stretches the square constellation into a rectangle, as illustrated in Fig. 2(c). After stretching, we propose the interleaver $\Pi=\left[\boldsymbol{e}_{1}, \boldsymbol{e}_{6}, \boldsymbol{e}_{3}\right.$, $\left.\boldsymbol{e}_{8}, \boldsymbol{e}_{2}, \boldsymbol{e}_{5}, \boldsymbol{e}_{4}, \boldsymbol{e}_{7}\right]$ and the same encoder $\mathbf{G}(\mathbf{s})$ from (5) that is

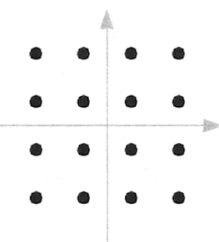

(a)

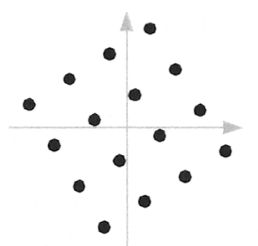

(b)

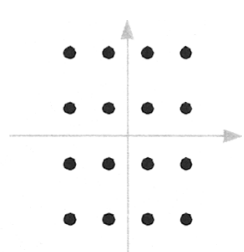

(c)
Fig. 2. 16-QAM (a); (b) rotated; (c) stretched. 
used in the Yuen-Guan-Tjhung code. Thus, in terms of the original information symbols, the proposed code for four transmit antennas is:

$$
\sqrt{\frac{2}{1+K^{2}}}\left[\begin{array}{rrrr}
K x_{1}^{R}+j x_{3}^{I} & K x_{2}^{R}+j x_{4}^{I} & x_{1}^{I}+j K x_{3}^{R} & x_{2}^{I}+j K x_{4}^{R} \\
-K x_{2}^{R}+j x_{4}^{I} & K x_{1}^{R}-j x_{3}^{I} & -x_{2}^{I}+j K x_{4}^{R} & x_{1}^{I}-j K x_{3}^{R} \\
x_{1}^{I}+j K x_{3}^{R} & x_{2}^{I}+j K x_{4}^{R} & K x_{1}^{R}+j x_{3}^{I} & K x_{2}^{R}+j x_{4}^{I} \\
-x_{2}^{I}+j K x_{4}^{R} & x_{1}^{I}-j K x_{3}^{R} & -K x_{2}^{R}+j x_{4}^{I} & K x_{1}^{R}-j x_{3}^{I}
\end{array}\right] \text { (7) }
$$

Proposition 1. The stretching parameter $K \in(0,1]$ that maximizes the coding gain (3) for the space-time code in (7) with QAM is $K=1 / \sqrt{2}$. The resulting asymptotic coding gain is $2 d_{\min }^{2} / 3$, where $d_{\min }$ is the minimum distance of the QAM alphabet $\mathcal{A}$.

Proof: Substituting from the definition of $\mathbf{G}(\mathbf{s})$ in (5), the asymptotic coding gain of (3) is:

$$
\begin{aligned}
\Gamma(K) & =\min \operatorname{det}\left((\mathbf{X}-\tilde{\mathbf{X}})^{*}(\mathbf{X}-\tilde{\mathbf{X}})\right)^{1 / 4} \\
& =\min \operatorname{det}\left[\begin{array}{lllll}
\alpha & 0 & \beta & 0 \\
0 & \alpha & 0 & \beta \\
\beta & 0 & \alpha & 0 \\
0 & \beta & 0 & \alpha
\end{array}\right]^{1 / 4} \\
& =\min \left(\alpha^{2}-\beta^{2}\right)^{1 / 2},
\end{aligned}
$$

where we have introduced $\alpha=\sum_{i=1}^{4}\left|\Delta s_{i}\right|^{2}=\sum_{i}\left|\Delta a_{i}\right|^{2}, \boldsymbol{\beta}=$ $2 \operatorname{Re}\left\{\Delta s_{1} \Delta s_{3}^{*}+\Delta s_{2} \Delta s_{4}^{*}\right\}=2 \sum_{i} \Delta a_{i}^{R} \Delta a_{i}^{I}, \quad \Delta s_{i}=s_{i}-\tilde{s}_{i}$, and $\Delta a_{i}=a_{i}-\tilde{a}_{i}$. The expressions for $\alpha$ and $\beta$ in terms of $\Delta a_{i}$ follow from $\mathbf{s}=\Pi(\mathbf{a})$. The minimization in (8) is over all nonzero vectors $\left[\Delta a_{1}, \ldots \Delta a_{4}\right]$. But

$$
\begin{aligned}
& \boldsymbol{\alpha}^{2}-\boldsymbol{\beta}^{2} \\
& =(\boldsymbol{\alpha}+\boldsymbol{\beta})(\boldsymbol{\alpha}-\boldsymbol{\beta}) \\
& =\left\{\sum_{i}\left(\left|\Delta a_{i}\right|^{2}+2 \Delta a_{i}^{R} \Delta a_{i}^{I}\right)\right\}\left\{\sum_{j}\left(\left|\Delta a_{j}\right|^{2}-2 \Delta a_{j}^{R} \Delta a_{j}^{I}\right)\right\} \\
& =\left\{\sum_{i}\left(\Delta a_{i}^{R}+\Delta a_{i}^{I}\right)^{2}\right\}\left\{\sum_{j}\left(\Delta a_{j}^{R}-\Delta a_{j}^{I}\right)^{2}\right\} .
\end{aligned}
$$

Because both factors in (9) are the sum of squares, the nonzero vector $\left[\Delta a_{1}, \ldots \Delta a_{4}\right]$ that minimizes the product will have only one nonzero element, say $\Delta a_{i}$, so that the gain (8) reduces to:

$$
\Gamma(K)=\min \left|\left(\Delta a_{i}^{R}\right)^{2}-\left(\Delta a_{i}^{I}\right)^{2}\right|,
$$

where now the minimization is over all nonzero error symbols $\Delta a_{i}$. But from (6) we can write:

$$
\Delta a_{i}=\sqrt{\frac{2}{1+K^{2}}}(K m+j n) d_{\min }
$$

for some pair of integers $(m, n) \in\{-\sqrt{M}+1, \ldots, \sqrt{M}-1\}^{2}$, where $d_{\min }$ is the minimum Euclidean distance of the (unstretched) alphabet $\mathcal{A}$. Plugging (11) into (10) yields the following expression for the coding gain, as a function of the parameter $K$ :

$$
\Gamma(K)=2 d_{\min }^{2} \cdot \min _{(m, n) \in \mathcal{M}} \frac{\left|K^{2} m^{2}-n^{2}\right|}{1+K^{2}},
$$

where we have introduced $\mathcal{M} \triangleq\{-\sqrt{M}+1, \ldots, \sqrt{M}-1\}^{2}-$ $\{(0,0)\}$ as the set of all possible integer pairs $(m, n)$, with the $(0,0)$ pair excluded.

The remaining problem is to find the value of $K$ that maximizes (12). We proceed in two steps: first, we establish the bound $\Gamma(K) \leq 2 d_{\min }^{2} / 3$; then, we show that $K=1 / \sqrt{2}$ achieves the bound with equality.

To establish the bound, let us introduce $\mathcal{L} \triangleq\{(1,0),(1,1)\} \subset$ $\mathcal{M}$. Because $\mathcal{L}$ is a subset of $\mathcal{M}$, it clearly follows that $\min _{\mathcal{M}}\{\cdot\} \leq \min _{\mathcal{L}}\{\cdot\}$, so that the coding gain of (12) can be bounded by:

$$
\begin{aligned}
\Gamma(K) & \leq 2 d_{\min }^{2} \cdot \min _{(m, n) \in \mathcal{L}} \frac{\left\lfloor K^{2} m^{2}-n^{2} \mid\right.}{1+K^{2}} \\
& = \\
2 d_{\min }^{2} \cdot \min & \left\{\frac{\left\lfloor K^{2}(1)^{2}-(0)^{2}\right\rfloor}{1+K^{2}}, \frac{\left.\mid K^{2}(1)^{2}-(1)^{2}\right\rfloor}{1+K^{2}}\right\} \\
& \leq 2 d_{\min }^{2} \cdot \max _{K}>0 \min \left\{\frac{K^{2}}{1+K^{2}}, \frac{1-K^{2}}{1+K^{2}}\right\} \\
& =\frac{2}{3} d_{\min }^{2} .
\end{aligned}
$$

The inequality of (13) follows from the fact that $g(K) \leq \max _{K>0}\{g(K)\}$ for any function $g(K)$ and for any $K>0$. The equality in (14) follows because, for $K>0$, the functions $K^{2} /\left(1+K^{2}\right)$ and $\left(1-K^{2}\right) /\left(1+K^{2}\right)$ are monotonically increasing and decreasing, respectively, so that the maximum of the minimum occurs at the intersection, namely at $K=1 / \sqrt{2}$.

The bound $\Gamma(K) \leq 2 d_{\min }^{2} / 3$ of (14) is in fact achievable with $K=1 / \sqrt{2}$, since from (12):

$$
\begin{aligned}
\Gamma(1 / \sqrt{2}) & =2 d_{\min }^{2} \cdot \min _{(m, n) \in \mathcal{M}} \frac{\left|m^{2} / 2-n^{2}\right|}{1+1 / 2} \\
& =\frac{2}{3} d_{\min }^{2},
\end{aligned}
$$

where the second equality follows from the fact that $\left|m^{2}-2 n^{2}\right|$ must be a nonnegative integer, and it cannot be zero because $\sqrt{2}$ is irrational; therefore, $\min _{(m, n) \in \mathcal{M}}\left|m^{2}-2 n^{2}\right|=1$.

We make two important remarks regarding the PAPR of the proposed code. First, stretching the alphabet according to (6) does not alter its PAPR. Indeed, it is easily verified that the stretched alphabet has the same PAPR as the original one, since:

$$
\begin{aligned}
\max _{\mathcal{A}}\left(\left|K x_{i}^{R}+j x_{i}^{I}\right|^{2}\right) & =\left(1+K^{2}\right)(\sqrt{M}-1)^{2}, \\
E\left(\left|K x_{i}^{R}+j x_{i}^{I}\right|^{2}\right) & =\left(1+K^{2}\right)(M-1) / 3 .
\end{aligned}
$$


Taking the ratio of these two yields $3(\sqrt{M}-1) /(\sqrt{M}+1)$, which is precisely the PAPR of the underlying QAM alphabet.

Second, our particular choice for coordinate interleaving does not alter the PAPR; this latter fact can be seen by observing that the elements of (7) all have the same PAPR as the stretched alphabet. Together, these two facts imply that the PAPR of the proposed code is identical to that of the underlying QAM alphabet, regardless of the value of $K$.

The proposed space-time code for three antennas is obtained by deleting the fourth column of $\mathbf{X}$ in (7). The proof that the space-time code obtained by deleting the fourth column is still fully diverse follows directly from Theorem 3 and Theorem 4 in [14]. For two antennas, an equivalent form of the Alamouti code [1] can be obtained by deleting columns three and four (or two and three) and setting $K=1$.

We will see in the next section that the proposed code is quasiorthogonal and single-symbol decodable.

\section{B. Single-Symbol ML Decoding of Proposed Code}

By conjugating two of its elements, the received vector in (1) can be rewritten as

$$
\left[\begin{array}{l}
y_{1} \\
y_{2}^{*} \\
y_{3} \\
y_{4}^{*}
\end{array}\right]=\left[\begin{array}{cccc}
h_{1} & h_{2} & h_{3} & h_{4} \\
h_{2}^{*} & -h_{1}^{*} & h_{4}^{*} & -h_{3}^{*} \\
h_{3} & h_{4} & h_{1} & h_{2} \\
h_{4}^{*} & -h_{3}^{*} & h_{2}^{*} & -h_{1}^{*}
\end{array}\right]\left[\begin{array}{l}
s_{1} \\
s_{2} \\
s_{3} \\
s_{4}
\end{array}\right]+\left[\begin{array}{c}
w_{1} \\
w_{2}^{*} \\
w_{3} \\
w_{4}^{*}
\end{array}\right]
$$

or

$$
\mathbf{y}=\mathbf{H s}+\mathbf{w}^{\prime} \text {. }
$$

The ML decision is the symbol vector $\mathbf{s}$ that minimizes

$$
\|\mathbf{y}-\mathbf{H s}\|^{2}=\|\mathbf{y}\|^{2}+\mathbf{s}^{*} \mathbf{H}^{*} \mathbf{H s}-2 \operatorname{Re}\left\{\mathbf{s}^{*} \mathbf{v}\right\},
$$

where we have introduced the vector $\mathbf{v}=\mathbf{H}^{*} \mathbf{y}$, which can be interpreted as the output of a filter matched to $\mathbf{H}$. Let $\eta=\sum_{i}\left|h_{i}\right|^{2}$ and $\rho=2 \operatorname{Re}\left\{h_{1} h_{3}^{*}+h_{2} h_{4}^{*}\right\}$. Then $\mathbf{H}^{*} \mathbf{H}$ is a circulant matrix whose first row is $[\eta, 0, \rho, 0]$, so that (19) reduces to:

$$
\begin{aligned}
\|\mathbf{y}-\mathbf{H s}\|^{2}=\|\mathbf{y}\|^{2} & +\eta \sum_{i}\left|s_{i}\right|^{2} \\
& +2 \rho \operatorname{Re}\left\{s_{1} s_{3}^{*}+s_{2} s_{4}^{*}\right\}-2 \operatorname{Re}\left\{\mathbf{s}^{*} \mathbf{v}\right\} .
\end{aligned}
$$

Already we can see that the symbols $s_{1}$ and $s_{3}$ can be decoded separately from $s_{2}$ and $s_{4}$. However, the decoding complexity reduces even further when we substitute $\mathbf{s}=\Pi(\mathbf{a})$ into (20), yielding:

$$
\|\mathbf{y}-\mathbf{H s}\|^{2}=\|\mathbf{y}\|^{2}+\sum_{i=1}^{4} f\left(z_{i} \mid a_{i}\right)
$$

where $f\left(z_{i} \mid a_{i}\right)=\eta\left|a_{i}\right|^{2}+\rho \operatorname{Im}\left\{a_{i}^{2}\right\}-2 \operatorname{Re}\left\{a_{i}^{*} z_{i}\right\}$,

where we have introduced $\mathbf{z}=\Pi^{-1}(\mathbf{v})=\left[v_{1}^{R}+j v_{3}^{R}\right.$, $\left.v_{2}^{R}+j v_{4}^{R}, \quad v_{3}^{I}+j v_{1}^{I}, \quad v_{4}^{I}+j v_{2}^{I}\right]$. Because each $f\left(z_{i} \mid a_{i}\right)$ depends on $\mathbf{z}$ and $\mathbf{a}$ only through $z_{i}$ and $a_{i}$, ML decoding reduces to separate decoding of the four transmitted symbols. Specifically, as illustrated in Fig. 3, the ML decision about $a_{i}$ (and ultimately $x_{i}$ ) can be found by minimizing the corresponding $f\left(z_{i} \mid a_{i}\right)$ metric of (22).

In Table I we compare the proposed code with other singlesymbol decodable codes in terms of decoding complexity and PAPR for four transmit antennas. We see that the PAPR of the proposed code is significantly smaller than that of the KhanRajan code [10], and that it has the same PAPR as the Su-Xia code [8] but at a reduced decoding complexity.

\section{NUMERICAL RESULTS}

In this section we compare the bit-error rate performance of the proposed space-time code with the Su-Xia code [8] and Yuen-Guan-Tjhung code [9] over a quasistatic Rayleigh-fading channel with additive Gaussian noise. To avoid clutter we do not include the Khan-Rajan code [10] in our comparison; we simply note that it performs comparably to the Yuen-GuanTjhung code with four transmit antennas, and slightly worse with three antennas. In Fig. 4 we show the bit-error rate performance for three antennas, while in Fig. 5 we show the performance for four antennas. Results are shown for 4-QAM, 16-QAM and 64-QAM Gray-coded alphabets.

Fig. 4 shows that, for three antennas, the proposed code performs only $0.5 \mathrm{~dB}$ worse than the Yuen-Guan-Tjhung code, and $0.6 \mathrm{~dB}$ worse than the Su-Xia code. For four antennas, the proposed code suffers a $0.7 \mathrm{~dB}$ and $0.8 \mathrm{~dB}$ loss compared with the Yuen-Guan-Tjhung code and Su-Xia code, respectively. The proposed code, however, has a lower decoding complexity than the Su-Xia code and maintains the same PAPR. The proposed code has a significantly lower PAPR than the Yuen-GuanTjhung code while suffering only a slight performance loss.

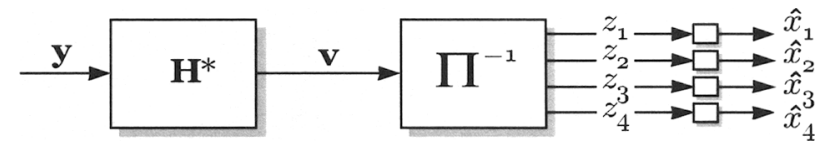

Fig. 3. The ML decoder for the proposed space-time code decomposes into a MF bank, a deinterleaver, and a bank of four independent complex-symbol quantizers.

Table I: A COMPARISON OF DECODING COMPLEXITY AND PAPR OF FOUR-ANTENNA SPACE-TIME BLOCK CODES.

\begin{tabular}{|l|c|c|c|c|}
\hline \multirow{2}{*}{\multicolumn{1}{|c|}{ Code }} & \multirow{2}{*}{$\begin{array}{c}\text { Number symbols } \\
\text { for joint detection }\end{array}$} & \multicolumn{3}{|c|}{ PAPR (dB) } \\
\cline { 3 - 5 } & & 4-QAM & 16-QAM & 64-QAM \\
\hline Su-Xia [8] & 4 & 0 & 2.55 & 3.68 \\
\hline Yuen et al.[9] & 2 & 1.61 & 4.16 & 5.29 \\
\hline Khan-Rajan [10] & 2 & 5.79 & 8.34 & 9.47 \\
\hline Proposed & 2 & 0 & 2.55 & 3.68 \\
\hline
\end{tabular}




\section{CONCLUSIONS}

We introduced a novel space-time block code for three and four transmit antennas that combines coordinate interleaving with constellation stretching. The stretching parameter was chosen analytically to maximize the coding gain. The resulting code has a quasiorthogonal structure that makes it singlesymbol decodable, meaning that the jointly maximumlikelihood decoder may be implemented using a bank of independent quantizers, one for each complex information symbol. The proposed codes for three and four antennas achieve full rate, full diversity, and single-symbol decodability without sacrificing the peak-to-average power ratio.

\section{REFERENCES}

[1] S. M. Alamouti, "A simple transmit diversity technique for wireless communications," IEEE J. SAC., vol. 16, pp. 1451-1458, Oct. 1998.

[2] V. Tarokh, H. Jafarkhani, A. Calderbank, "Space-Time Block codes from orthogonal designs," IEEE Trans. IT, 45:5, pp.1456-, July 1999.

[3] H. Jafarkhani, "A quasi-orthogonal space-time block code," IEEE Trans. Commun., vol. 49, no. 1, pp. 1-4, Jan. 2001.

[4] O. Tirkkonen, A. Boariu, and A. Hottinen, "Minimal nonorthogonality rate 1 space-time block code for 3+ Tx antennas," in Proc. IEEE 6th Int. Symp. Spread-Spectrum Techniques and Applications (ISSSTA 2000), Sept. 2000, pp. 429-432.

[5] C. B. Papadias and G. J. Foschini, "Capacity-Approaching SpaceTime Codes for Systems Employing Four Transmitter Antennas," IEEE Trans. on Inf. Theory, vol. 49, No. 3, pp. 726-732, Mar. 2003.

[6] O. Tirkkonen, "Optimizing space-time block codes by constellation rotations," Proc. Finnish Wireless Commun. Workshop FWCW'01, Finland, pp. 59-60, Oct. 2001.

[7] N. Sharma and C. Papadias, "Improved quasi-orthogonal codes through constellation rotation," IEEE T-COM. 51:3, pp. 332-, 2003.

[8] W. Su and X.-G. Xia, "Signal constellations for quasi-orthogonal space-time block codes with full diversity," IEEE Trans. Inf. Theory, vol. 50, pp. 2331-2347, Oct. 2004.

[9] C. Yuen, Y. L. Guan, and T. T. Tjhung, "Quasi-Orthogonal STBC With Minimum Decoding Complexity," IEEE Trans. Wireless Commun., vol. 4, no. 5, pp. 2089-2094, Sep. 2005.

[10] M. Z. A. Khan and B. S. Rajan, "Single-Symbol Maximum Likelihood Decodable Linear STBCs," IEEE Trans. on Inf. Theory, vol. 52, no. 5, pp. 2062-2091, May 2006.

[11] A. Jayalath and C. Tellambura, "Peak-to-average power ratio of IEEE 802.11a PHY layer signals," in Proc. International Symposium on DSP for Communication Systems, Australia, Jan. 2002, pp. 31-36.

[12] P. Marsch, W. Rave and G. Fettweis, "Quasi-Orthogonal STBC using stretched constellations for low detection complexity," WCNC, pp. 757-761, March. 2007.

[13] P. Dayal and M. K. Varanasi, "Maximal Diversity Algebraic SpaceTime Codes With Low Peak-to-Mean Power Ratio," IEEE Trans. on Inf. Theory, vol. 51, No. 5, pp. 1691-1708, May 2005.

[14] C. Yuen, Y. L. Guan, and T. T. Tjhung, "Quasi-orthogonal STBC with minimum decoding complexity: Further results," in Proc. Wireless Communications and Networking Conf. (WCNC), New Orleans, 2005, pp. 483-488.

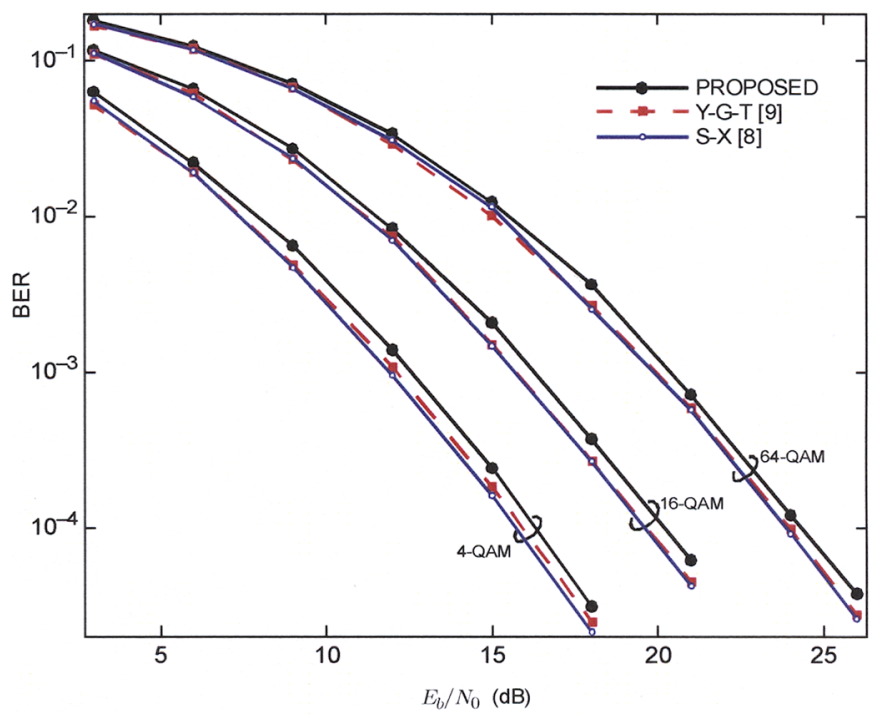

Fig. 4. Performance for three transmit antennas.

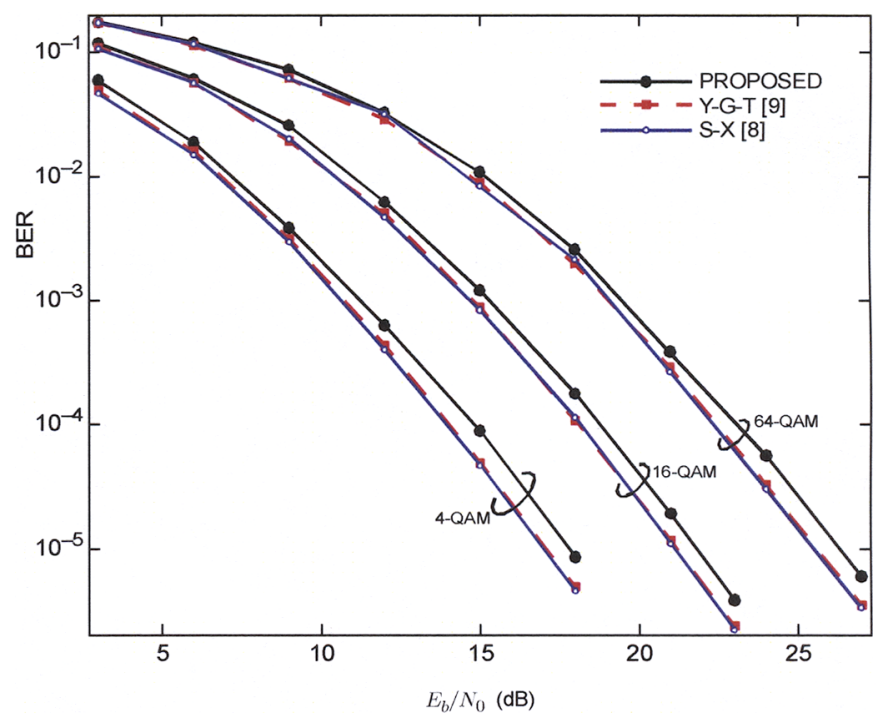

Fig. 5. Performance for four transmit antennas. 